\title{
Рынок и технологии в силовой электронике: отчеты Yole Développement
}

\author{
Ю. Ковалевский, Е. Суворов
}

\begin{abstract}
Любое электронное устройство требует электропитания. Поэтому сложно назвать сектор разработки и производства электронных устройств, в котором не проявлялся бы интерес к развитию технологий и состоянию рынка силовой электроники. Росту этого интереса способствуют и такие мировые тренды, как развитие электрического транспорта, "умные электросети", робототехника, а также телекоммуникации 5С и Интернет вещей, где также существуют свои специфические требования к электропитанию.

Чтобы предоставить специалистам отрасли возможность быть в курсе текущего положения вещей и представлять себе как общую картину, так и детали рынка и технологий в области электроники, Yole Développement - одна из ведущих аналитических компаний отрасли - проводит различные исследования, в том числе и в секторе силовой электроники. Нескольким отчетам, выпущенным компанией по результатам таких исследований в 2018 году, посвящена данная статья.
\end{abstract}

$\mathrm{K}$ омпания Yole Développement недавно отметила свое двадцатилетие: она была образована в 1998 году. Сейчас она входит в состав Yole Group of Companies - группы компаний, выросшей на ее основе, которая предоставляет консалтинговые и информационные услуги в области маркетинга, технологий и стратегий, а также консультационные услуги в финансовой сфере.

Компания специализируется на таких областях, как МЭмС и датчики, рч-электроника, медицинские и биотехнологии, фотоника и оптоэлектроника, полупроводниковые материалы и технологии, память, твердотельное освещение и многое другое, включая такое важное и активно развивающееся направление, как силовая электроника.

Общая картина рынка силовой электроники приводится в отчете "Состояние индустрии силовой электроники" [1], выпущенном в августе 2018 года. Отчет, насчитывающий более трех с половиной сотен страниц, основан на данных компаний со всего мира, которые включают в себя не только производителей компонентов и устройств силовой электроники, но и их потребителей из различных отраслей. Среди этих компаний такие, как ABB, Analog Devices, BMW Group, Bosch, Continental, Cree, Delphi, Fuji Electric, General Electric, Hitachi, Huawei, Infineon, Intel, Microchip, Microsemi, Mitsubishi Electric, Nissan, NXP, ON Semiconductor, Panasonic, Qualcomm, Renault, Renesas, Siemens, STMicroelectronics, TDK, Tesla, Toshiba, Toyota, TSMC, Vishay, Volkswagen и многие другие. В этом списке неслучайно присутствует целый ряд крупных автомобильных производителей: в отчете теме электрического и гибридного транспорта посвящен отдельный раздел. Также с этого направления начинается раздел, озаглавленный "Общие тренды». Другие области, рассматриваемые в данном разделе, включают в себя ветроэлектрогенерацию, железнодорожный транспорт, источники бесперебойного питания, электроприводы, фотовольтаику, потребительскую электронику, аудио- и видеоаппаратуру, бытовую технику, медицинское применение, вычислительную технику и хранение данных, системы накопления энергии и зарядные устройства постоянного тока.

В отчете состояние рынка силовой электроники рассматривается как в целом, так и по таким направлениям, как полупроводниковые пластины, MOSFET, IGBT, ИС, модули и инверторы. Также рассматриваются технические вопросы, связанные, в частности, с обеспечением теплового режима и ростом интеграции в преобразователях питания. Отдельные разделы посвящены пассивным и широкозонным компонентам, а также финансовому анализу компаний, работающих в области силовой электроники, и слияниям и поглощениям на рынке. Некоторые из этих разделов включены в отчет впервые. Кроме того, были обновлены данные и прогнозы рынков силовой электроники на уровне пластин, дискретных компонентов и модулей, включая IGBT, MOSFET и ИС, и инверторов на период с 2017 по 2023 год.

По данным, приведенным в отчете, в секторе силовых полупроводников в 2017 году объем рынка достиг 32,7 млрд долл., а в секторе IGBТ рост составил 11,7\%. Кроме того, указывается, что растет интерес к вопросам обеспечения теплового режима и применения в тяжелых условиях эксплуатации, компоненты на основе SiC и GaN будут 


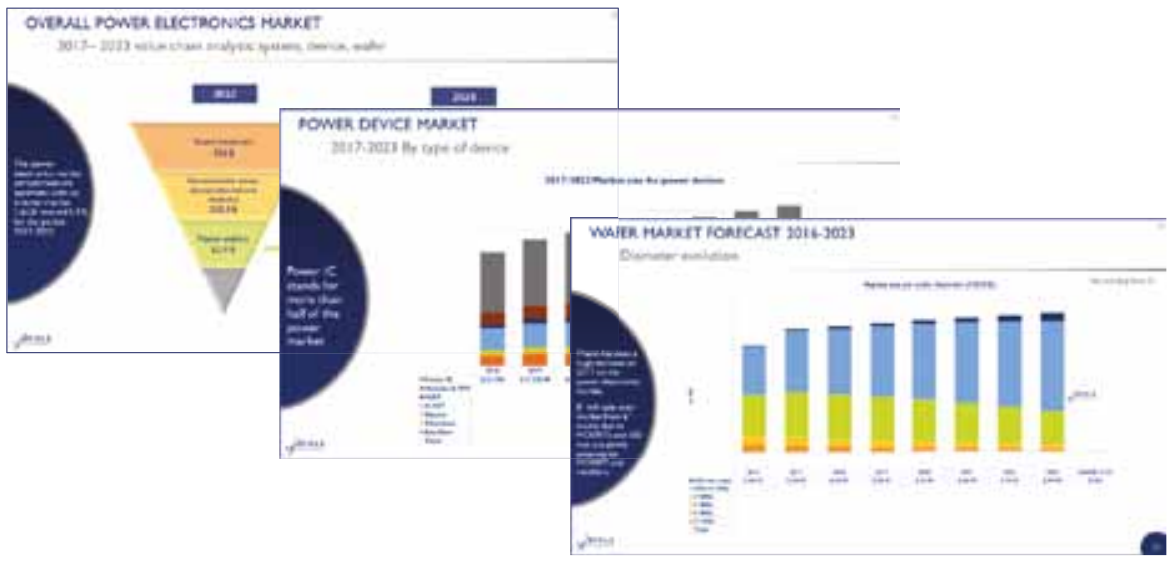

Примеры прогноза рынка из отчета «Состояние индустрии силовой электроники" [2]

отбирать долю рынка у кремниевых приборов, а силовые модули, по ожиданиям аналитиков Yole Développement, заберут себе часть рынка дискретных компонентов. Рынок фотовольтаики, спад которого начиная с 2018 года прогнозируется в отчете, предположительно, в дальнейшем будет постепенно восстанавливаться. Эти сведения обозначены, как ключевые выводы отчета [2], который, как уже говорилось, предоставляет возможность взглянуть на состояние рынка силовой электроники, его драйверов и технологических фокусов в целом.

Наиболее важным направлениям в силовой электронике посвящены отдельные отчеты компании Yole Développement, в которых данные области рассматриваются более подробно и которые дополнены информацией, особенно актуальной для данного конкретного сектора.

Так, в отчете «Силовая электроника для электрических и гибридных транспортных средств" [3], выпущенном в апреле 2018 года, помимо обновленных сведений о рынке и его прогнозов до 2023 года, анализа стоимости силовой электроники для электрического и гибридного транспорта на системном уровне и позиций компаний, задействованных в развитии технологий для автомобильной промышленности, в том числе на базе карбида кремния, приводятся сведения, касающиеся новых систем с напряжением 48 В, а также новых продуктов, появившихся с 2016 года. Кроме того, в отчете рассматривается развитие китайского рынка в данной области до 2030 года.

Вопросам рынка силовых ИС также посвящен отдельный отчет: "Введение в рынок силовых интегральных схем" [4] от октября 2018 года, в котором содержатся поквартальные прогнозы и сведения о долях рынка в данном сегменте, доступные в формате Excel. Отчет включает обновленную информацию об ис для организации электропитания и их применении. Данные о рынке и ключевыхпримененияхприводятся по пяти основным направлениям - автомобильной отрасли, потребительским устройствам, вычислительной технике, телекоммуникациям и промышленной электронике. Кроме того, информация также разделена по типам ИС для организации питания, включая импульсные стабилизаторы, контроллеры, линейные стабилизаторы, стабилизаторыс малым падением напряжения, источники опорного напряжения, многоканальные ИС для источников питания и др. [5].

Отчет позволяет не только получить общее представление о конкурентной среде на рынке силовых ИС и данные, охватывающие более 40 секторов по конечному применению, но и понять рыночную динамику в этой сфере.

Как уже говорилось, силовые модули, по ожиданиям специалистов Yole, могут забрать часть рынка дискретных компонентов. В 2017 году рынок силовых модулей на основе IGBT показал рост 18,1\% в сравнении с предыдущим годом, и хотя в отношении других типов подобных изделий, таких как модули на основе MOSFET, имело место небольшое снижение, в целом объем рынка силовых модулей в 2017 году составил 3,54 млрд долл., демонстрируя ежегодный рост на уровне 11,3\%. Ожидается, что к 2023 году этот рынок превысит показатель 5,5 млрд долл., что открывает большие возможности для развития бизнеса в области материалов для корпусирования силовых модулей [6]. Этот вопрос нашел отражение в отчете «Корпусирование силовых модулей: рынок материалов и технологические тренды", вышедшем в июле 2018 года [7].

Следует отметить, что наряду с анализом рынка силовых модулей и материалов для них в данном отчете приводятся сведения о том, как решаются характерные для

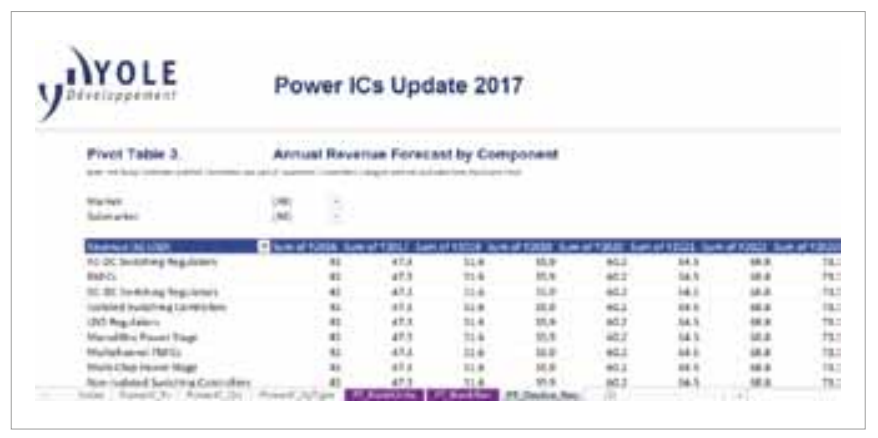

Пример представления данных по типам ИС в отчете «Введение в рынок силовых интегральных схем» [5] 
этого направления технические проблемы, какие существуют в этом отношении тренды. В частности, рассматриваются такие темы, как термомеханические характеристики, корпусирование, выполнение межсоединений, крепление кристаллов, герметизация и др.

Также в отчете приводится анализ бизнес-моделей и цепочек поставок в области силовых модулей.

Еще одно многообещающее направление в силовой электронике - изделия на основе карбида кремния. SiC-транзисторы находят все более широкое применение в различных областях. По прогнозам Yole Développement, объем рынка силовых полупроводников на основе SiC к 2023 году достигнет величины более 1,5 млрд долл., при этом совокупный темп годового роста (CAGR) в периоде с 2017 по 2023 год будет составлять 31\%. В настоящее время развитие данного рынка все еще связано с применением диодов в устройствах коррекции коэффициента мощности и в фотовольтаике, однако специалисты Yole ожидают, что через несколько лет драйвером рынка SiC-компонентов станут транзисторы. Это будет обусловлено отчасти улучшением характеристик и надежности SiC-транзисторов в сравнении с первым поколением данных изделий, благодаря чему заказчики будут более уверенно использовать их в своих изделиях [8]

Развитие рынка силовой электроники на основе карбида кремния отражено в отчете Yole Développement «Силовая электроника на SiC: материалы, устройства и применение" [9] от июля 2018 года. В отчете приводятся обновленные сведения об объеме рынка дискретных диодов, диодов в гибридных модулях, дискретных транзисторов, модулей на основе карбида кремния, бескорпусных диодов и транзисторов. Новые сведения приведены и о статусе игроков рынка. Кроме того, в отчете описывается проникновение SiC-технологий в электрический и гибридный транспорт и в фотовольтаику - сучетом влияния новой политики Китая. Уделяется внимание и рынку эпитаксиальных пластин для SiC-компонентов. Вопросам надежности и ландшафту интеллектуальной собственности материалов и компонентов в области SiC-технологий посвящены новые разделы отчета.

Еще один отчет Yole Développement, выпущенный компанией в 2018 году - «Силовая электроника на GaN: эпитаксия, устройства, применение и технологические тренды" [10] - посвящен другому многообещающему материалу, обладающему большими перспективами в области силовых устройств. Хотя рынок силовой электроники на основе нитрида галлия остается очень малым в сравнении с рынком кремниевых силовых устройств, совершенно очевидно, что данный материал обладает значительными техническими преимуществами в сравнении с традиционным применением кремния в транзисторах MOSFET.
В отчете содержится анализ проникновения GaN-технологий в различные области, такие как источники питания, фотовольтаика, электрический и гибридный транспорт, бесперебойное питание, а также лидары и электропитание для беспроводных технологий. Помимо этого, приводится обзор передовых силовых компонентов на основе GaN с их описанием. Ландшафт отрасли силовой электроники на основе нитрида галлия, приведенный в отчете, охватывает цепочку от эпитаксиальных структур и разработки компонентов до изготовления изделий.

И безусловно, в отчете приводятся данные о состоянии и динамике рынка, а также прогноз до 2023 года.

Глубокий анализ огромного количества данных о рынке и технологических трендах, отраженный в отчетах Yole Développement, позволяет быть в курсе самых современных тенденций и идти в ногу со временем как при стратегическом планировании, так и при выборе конкретных технических решений в новых разработках, что особенно важно, когда речь идет об областях, отличающихся высокой динамикой, к каковым, безусловно, сегодня относится силовая электроника.

\section{ЛИТЕРАТУРА}

1. Status of the Power Electronics Industry 2018. https://www.i-micronews.com/products/status-of-the-powerelectronics-industry-2018/

2. https://yole-i-micronews-com.osu.eu-west-2.outscale.com/ uploads/2019/02/YD18033-Status_of_the_Power_Electronics_ Industry_2018_August_2018_Sample.pdf

3. Power Electronics for EV/HEV 2018. https://www.i-micronews. com/products/power-electronics-for-ev-hev-2018/

4. Introduction to the power IC market 2018. https://www.i-micronews.com/products/introduction-to-thepower-ic-market-2018/

5. https://yole-i-micronews-com.osu.eu-west-2.outscale.com/ uploads/2018/10/YD18040_Introduction_to_the_power_IC Market_October_2018_sample.pdf

6. https://yole-i-micronews-com.osu.eu-west-2.outscale.com/ uploads/2019/01/YD18032_Power_Module_packaging_ July2018_Yole_Flyer.pdf

7. Power Module Packaging 2018: Material Market and Technology Trends. https://www.i-micronews.com/products/power-modulepackaging-2018-material-market-and-technology-trends/

8. https://yole-i-micronews-com.osu.eu-west-2.outscale.com/ uploads/2019/01/YD18027_Power_SiC_2018_Materials_ Devices_Applications_July2018_Yole_Flyer.pdf

9. Power SiC 2018: Materials, Devices and Applications. https://www.i-micronews.com/products/powersic-2018-materials-devices-and-applications/

10. Power GaN 2018: Epitaxy, Devices, Applications and Technology Trends. https://www.i-micronews.com/products/powergan-2018-epitaxy-devices-applications-and-technology-trends/ 


\section{АНАЛИТИЧЕСКИЕ ОТЧЕТЫ 2018-2019}

МЭМС И ААТИИКИ

- Coctonane orpocna MJMC 2019

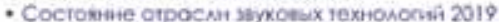

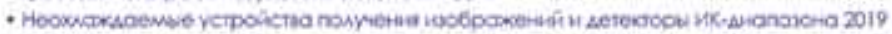

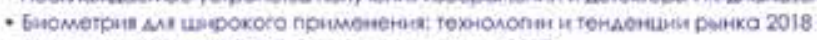

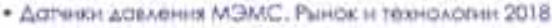

- Aarnaku roso н yocrinu 2016

\section{РЧ-УСТРОЙСТВА И ТЕХНОАОГИИ}

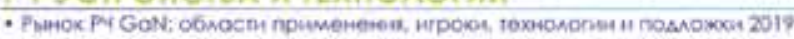

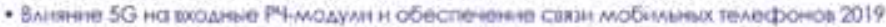

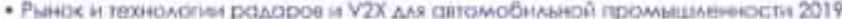

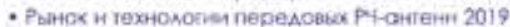

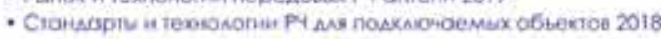

ФOTOHИKА И OПTОЗАЕКTPOHИKA

- Фorouhas usierpanthe crems 2019

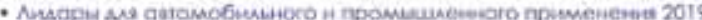

- Креминевan фотоника 2018

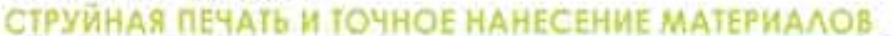

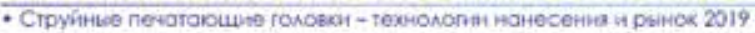

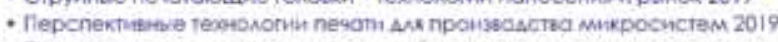

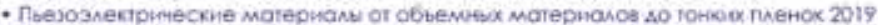

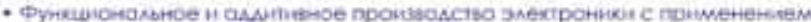

crpyйнoй nergin 2018

ПОАУПРОВОАНИКОВОЕ ПРОИЗВОАСТВО

- Honoumenpuhtoras nitorpochis 2019

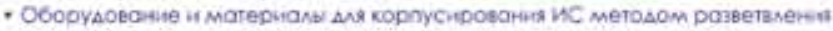

(Fon-out] 2019

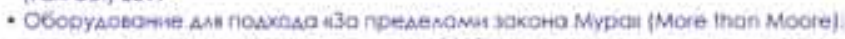

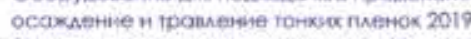

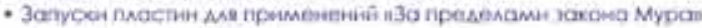

fMote fitan Moorel 2018

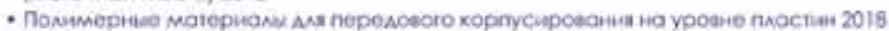

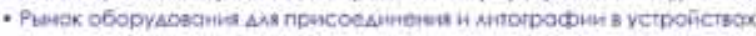

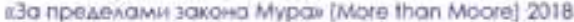

\section{ПAMSHF}

- Coctovene 6maneca nammtu 2019

- MRAM-Texionora и бumec 2019

- Пlepcnekrienar aнeprohejosucuman namuti 2018

ПОАУПРОВОАНИКОВЫЕ СОЕАИНЕНИЯ

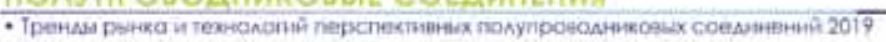

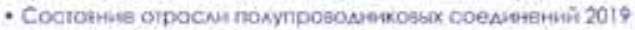

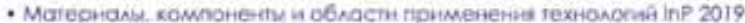

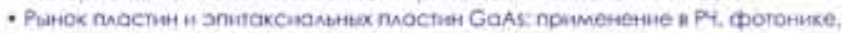

caetoniosax 14 dotosombranke 2018

\section{IEPEAOBOE XOPПYСИРОВАНИЕ ИС}

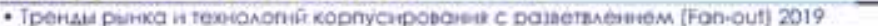

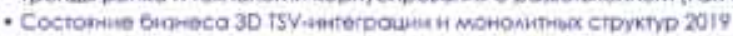

- Пepenouve Py CaK are mócunbrax tenedpanos 2019

- Cocionate nepenoeoro copmyorpotamia 2019

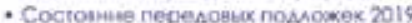

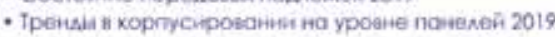

- P4-oictems is xoprityce in masepnara dus SG 2019

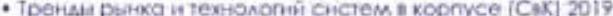

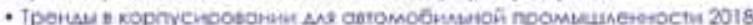

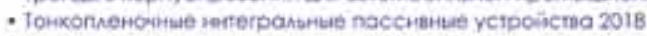

ПЕРЕАОВОЕ КОРПУСИРОВАНИЕ ИС

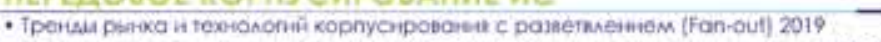

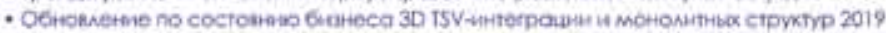

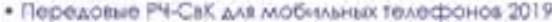

- Coctohine nepesovono eophycipotamia 2019

- Coctonue nepeasura nomosex 2019

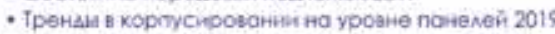

- P4-сnстемы a kopriyce it marephana A4 BG 2019

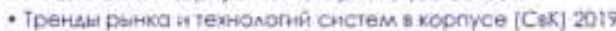

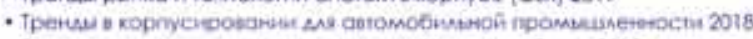

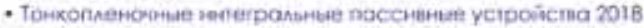

AMCחREH

- Hoboe nokomerbue 3D-auchnees 2019

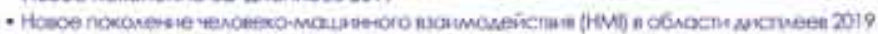

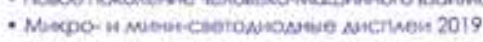

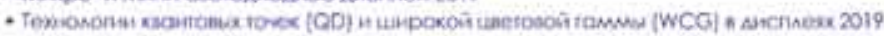

ПОЛУЧЕНИЕ ИЗОБРАЖЕНИЙ

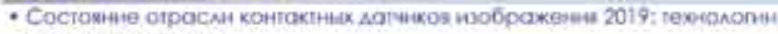

ифаунари-бивес

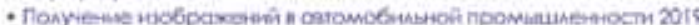

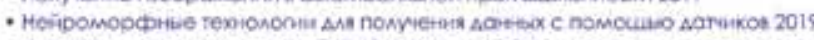

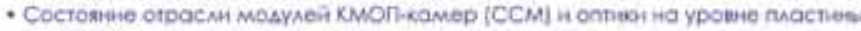

(WLO) 2018

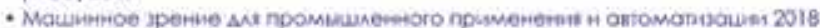

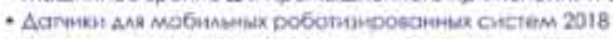

ВИЗУААИЗАЦИЯ В МЕАИЦИНЕ И БИОФОТОНИКА

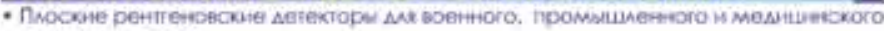
npimetitesers 2019

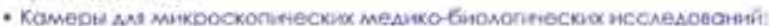

aranse piarika h тexiononsi 2019

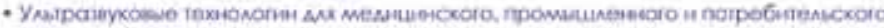
mpumeriesses 2018

\section{МИКРОФАКИАИК А}

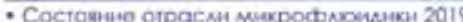

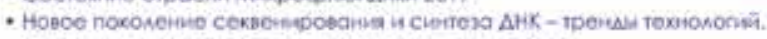

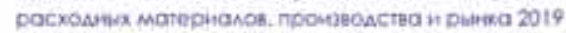

- Puн

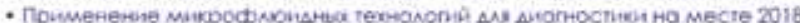

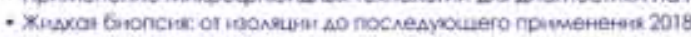

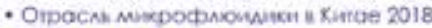

БHOTEXHOАOГИИ

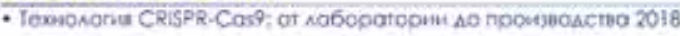

БИОМЗМС И MИКРОСКСТЕМЫ МЕАИЦИНСКОГО НАЗНАYЕНИЯ

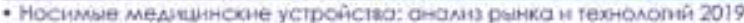

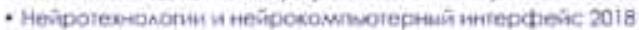

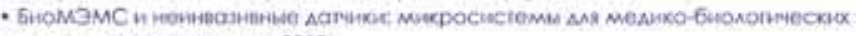

hayk in uponocoxpanesen 2018

ИНФОРМАНИОННЫЕ СИСТЕМЫ И ТЕХНОЛОГИИ

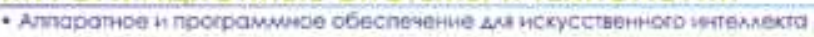

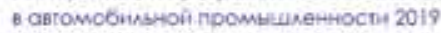

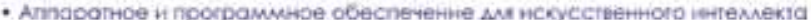

E notpebutentckic yctpoictecax 2019

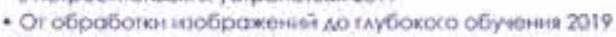

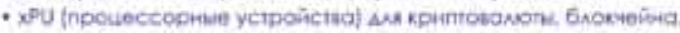

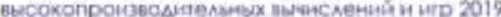

\section{СИАОВАЯ ЭАЕКТРОНИКА}

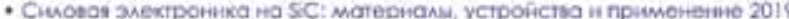

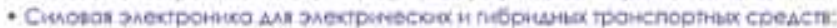

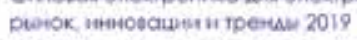

- Coctobete onpocur ciuonoñ suesipominer 2019

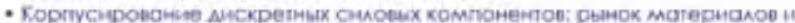

Texmonongueckue iperau 2019

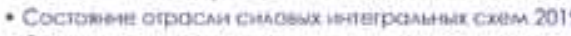

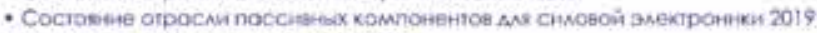

- Cocromsae orpochas uheepropos 2019

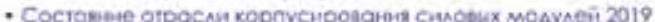

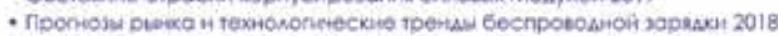

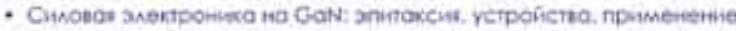

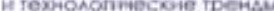

AКХУМУАЯTOPЫ И УПРАВАЕНИЕ ПИТАНИЕМ

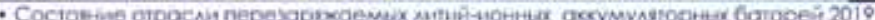

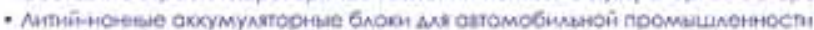

и стаLinohapmoto xponents כHeprin 2019

TBEPАOTEABHOE OCBEUЕНИE

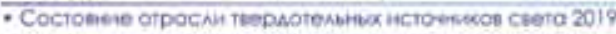

- Nosepe topuesoro ves raenus 2019

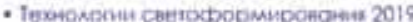

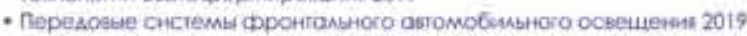

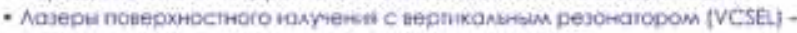

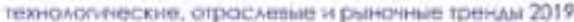

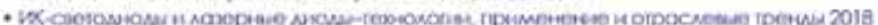

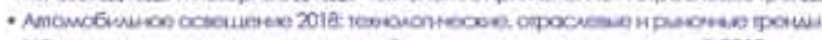

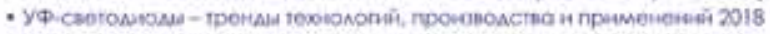

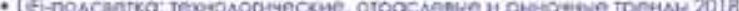




\section{gikn Turkey \\ Discover}

INNOPROM the potential
8-11 июля 2019, Екатеринбург МВЦ «Екатеринбург-ЭКспО;

\section{ИННОПРОМ}

МЕЖДУНАРОДНАЯ

ПРОМЫШЛЕННАЯ ВЫСТАВКА

СТРАНА-ПАРТНЕР:

\section{ТУРЕЦКАЯ РЕСПУБЛИКА}

TEMA:

\section{ЦИФРОВОЕ ПРОИЗВОДСТВО: ИНТЕГРИРОВАННЫЕ РЕШЕНИЯ}

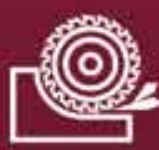

ИННОПРОМ МЕТАЛЛООБРАБОТКА

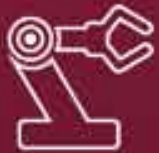

ИНДУСТРИАЛЬНАЯ АВТОМАТИЗАЦИЯ

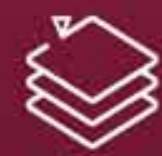

АДДИТИВНЫЕ технологии

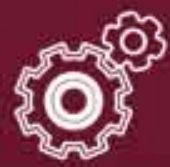

МАШИНОСТРОЕНИЕ И ПРОИЗВОДСТВО КOMПOHEHTOB

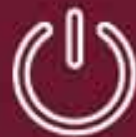

TEXнологИИ АЛЯ ЭНЕРГЕТИКИ
- 600 индустриальных компаний-экспонентов

- 46000 уникальных посетителей

из 107 стран мира

- более 160 деловых мероприятий 